\title{
Composite Correlation Filters for Detection of Geometrically Distorted Objects Using Noisy Training Images
}

\author{
Pablo M. Aguilar-González and Vitaly Kober \\ Department of Computer Science, Centro de Investigación Científica y de Educación \\ Superior de Ensenada, \\ Carretera Ensenada-Tijuana No. 3918, Zona Playitas, C.P. 22860, \\ Ensenada, B.C., México \\ \{paguilar, vkober\}@cicese.mx \\ http://www.cicese.edu.mx/
}

\begin{abstract}
Correlation filters for object detection use information about the appearance and shape of the object of interest. Therefore, detection performance degrades when the appearance of the object in the scene differs from the appearance used in the filter design process. This problem has been approached by utilizing composite filters designed from a training set containing known views of the object of interest. However, common composite filter design is usually carried out under the assumption that the ideal appearance and shape of the target are known. In this work we propose an algorithm for composite filter design using noisy training images. The algorithm is a modification of the class synthetic discriminant function technique that uses arbitrary filter impulse responses. Furthermore, filters can be adapted to achieve a prescribed discrimination capability for a class of backgrounds if a representative sample is known. Computer simulation results obtained with the proposed algorithm are presented and compared with those of common composite correlation filters.
\end{abstract}

Keywords: correlation filters, pattern recognition, composite filters.

\section{Introduction}

Correlation filters have been extensively used for pattern recognition to solve the problems of object detection and location estimation [1 23]. The task of object detection can be solved by searching for the highest peaks in the correlation filter output. The task of location estimation is then solved by taking the coordinates of those peaks as estimates of the true location of the object in the input scene.

The detection performance of correlation filters can be evaluated in terms of discrimination capability (DC) and probability of false alarms [4]. Location accuracy can be characterized by the variance of location errors [5]6. Correlation filers are designed to optimize one or several performance criteria for a chosen scene model. Two signal models are commonly used: additive and nonoverlapping. In 
the additive signal model a target is added to a noisy background. In the nonoverlapping model an opaque target is placed over a background and sensor noise is added to the whole scene. Optimizing the signal-to-noise ratio criterion for the additive model yields the Matched Filter [7]. Minimizing the probability of false alarms leads to the Optimal Filter (OF) [4]. For the nonoverlapping signal model the Generalized Matched Filter and the Generalized Optimum Filter (GOF) optimize the SNR and peak-to-output energy (POE) criteria, respectively [8].

Recently, generalizations of the OF and the GOF filters were proposed for a signal model that takes into account the presence of noise in the reference images used for filter design 910 . When the reference image is corrupted by additive noise and detection is carried out in an input scene that is nonoverlapping the signal model is referred to as the Additive-Nonoverlapping model (AN) 10.

Correlation filters can be characterized as appearance-based techniques, that is, they use information about the expected appearance and shape of a target to perform target detection. However, detection performance degrades rapidly as the appearance of the object in the scene differs from the appearance used to design a filter 1112. Appearance changes can be due to geometric distortions such as scale changes, in-plane and out-of-plane rotations; blurring and corruption by noise, and view changes of three-dimensional objects in two-dimensional images. Synthetic Discriminant Functions (SDF) were proposed to take into account multiple possible views of a target in a single correlation operation [13. SDF filters are designed to satisfy hard constraints for the correlation peak. Since only the intensity of the correlation peak is controlled, large sidelobes may appear in the rest of the correlation plane. Minimum Average Correlation Energy (MACE) filters were proposed to minimize the average energy in the filter output yielding a sharp correlation peak in the presence of the target and output close to zero in the background area [14. Maximum Average Correlation Height $(\mathrm{MACH})$ filters were proposed to improve the generalization capability of composite filters [15]. Composite filters can be designed to achieve a prescribed DC value using an adaptive approach. Instead of designing filters to have good performance across an image ensemble, adaptive filters are optimized for a class of backgrounds given by a representative example [16 17 18.

When degradations of the target and input scene can be modeled as a convolution with a linear filter, a priori information about the degradation function can be incorporated into the filter design process [1920].

However, composite correlation filter techniques assume that noiseless training images are available. In practice, the appearance of a target may be given in a noisy reference image or embedded in a complex background. In this paper, we present the a algorithm for composite correlation filter design using images corrupted by additive noise. Adaptive composite filters are designed using a modified SDF algorithm that combines the impulse response of multiple optimum filters as well as matched filters for patterns to reject. The performance of filters obtained with the proposed algorithm is compared with that of classical composite filters in terms of discrimination capability. 


\section{Composite Correlation Filter Algorithm}

In this section an algorithm for composite correlation filter design is presented. Filters are composed as a linear combination of optimum filter impulse responses designed from noisy training images. Using an example background an iterative adaptation procedure is used to guarantee a desired discrimination capability. The example background can be given as a realization of a random process or as a sample image. The nonoverlapping signal model is used for the input scene and the additive signal model is used for the training images. Throughout this section one-dimensional notation is used for simplicity. The same notation is used for a random process and its realization.

The $i$-th training image $r^{i}(x)$ is modeled as

$$
r_{i}(x)=t_{i}(x)+n_{i}^{r}(x),
$$

where $t_{i}(x)$ is the appearance of the target, and $n_{i}^{r}(x)$ is the additive noise present in the training image. The input scene $s(x)$ contains a nonoverlapping background as well as additive noise; formally, it is modeled as follows:

$$
s(x)=t_{i}(x)+b(x) w_{i}(x)+n^{s}(x),
$$

where $w_{i}(x)$ is the inverse region of support for $i$-th view of the target, $b(x)$ represents the nonoverlapping background, treated a realization of a stationary random process with mean $\mu$ and $n^{s}(x)$ is the additive sensor noise. The frequency response for the optimum filter can take two forms; which of the two forms achieves the best performance depends on the first and second order statistics of the target and nonoverlapping background [10. The optimum filter for each training image is designed using the algorithm given in Ref. [10]. The estimation of the composite target for each view is denoted by

$$
\hat{t}_{i}(x)=g_{i}(x) \bullet r_{i}(x)+\mu \hat{w}_{i}(x),
$$

where $g_{i}(x)$ denotes the impulse response of the Wiener filter designed to denoise the $i$-th training image, $\bullet$ denotes the convolution operation and $\hat{w}_{i}(x)$ denotes the estimation of $w_{i}(x)$ obtained from $r_{i}(x)$.

Let $h_{i}(x)$ denote the impulse response of the optimum filter for the $i$-th training image. A SDF filter can be composed as a linear combination of the set $\left\{h_{i}(x), i=1,2, \ldots, k\right\}$, where $k$ is the number of views of the target known a priori. The coefficients of the linear combination are chosen to satisfy output constraints for each training image. Let $\left\{p_{i}(x), i=1,2, \ldots, m\right\}$ denote the set of patterns to reject. The set of $k+m$ training patterns is denoted by $\mathbf{S}=\left\{\hat{t}_{1}(x), \ldots, \hat{t}_{k}(x), p_{1}(x), \ldots, p_{m}(x)\right\}$, where $t_{i}(x)$ is the estimation of the composite the target corresponding to the $i$-th view of the target. Let $\mathbf{H}=\left\{h_{1}(x), \ldots, h_{k}(x), p_{1}(x), \ldots, p_{m}(x)\right\}$ denote the set of optimum filter impulse responses and patterns to reject. The desired filter will be composed as

$$
h_{\mathrm{SDF}}(x)=\sum_{i=1}^{k} \alpha_{i} h_{i}(x)+\sum_{i=k+1}^{k+m} \alpha_{i} p_{i-k}(x) .
$$


Let $\mathbf{R}$ denote a matrix whose columns are the vector version of each element of $\mathbf{H}$. Let $\mathbf{a}=\left[\alpha_{1}, \ldots, \alpha_{k+m}\right]^{T}$ denote the vector of coefficients, where ${ }^{T}$ denotes transpose. Using vector-matrix notation, Eq. (4) can be rewritten as

$$
\mathbf{h}_{\mathrm{SDF}}=\mathbf{R} \mathbf{a} \text {. }
$$

The output constraints are given by the vector

$$
\mathbf{u}=[\underbrace{1, \ldots, 1}_{k \text { ones }}, \underbrace{0, \ldots, 0}_{m \text { zeros }}]^{T} .
$$

Let $\mathbf{Q}$ denote the matrix whose columns are the vector versions of the elements of $\mathbf{S}$. Satisfying the desired output constraints requires the following equation to hold:

$$
\mathbf{u}=\mathbf{Q}^{+} \mathbf{h}_{\mathrm{SDF}},
$$

where ${ }^{+}$denotes conjugate transpose. Using Eqs. (5) and (7), the solution for $\mathbf{h}_{\mathrm{SDF}}$ is given by

$$
\mathbf{h}_{\mathrm{SDF}}=\mathbf{R}\left[\mathbf{Q}^{+} \mathbf{R}\right]^{-1} \mathbf{u} .
$$

Using Eq. (8), the intensity of the correlation peak should be close to unity for true class objects and close to zero for rejected patterns. However, there is no explicit control over the correlation plane, there may be large sidelobes in the background area. To reduce the probability of false alarms, an adaptive procedure is used to reduce the intensity of the correlation output for a representative background. In each step, the filter is correlated with the sample background. A synthetic pattern to reject is taken from the background at the coordinates of the largest peak. The DC metric is used to characterize the ability of a filter to discriminate the object of interest against unwanted objects of similar appearance. The DC is formally defined as 21]

$$
D C=1-\frac{\left|C_{\max }^{b}\right|^{2}}{\left|C_{\max }^{t}\right|^{2}},
$$

where $C_{\max }^{b}$ and $C_{\max }^{t}$ are the maximum values in the correlation output over the area the background and the target, respectively. Values of the DC close to unity, indicate a good capacity to discriminate the target against unwanted objects. Negative values of the DC indicate a failure to detect the target.

A diagram of the proposed algorithm is shown in Fig. 1 The algorithm can be summarized as follows:

1. Using Eq. (8), create an SDF filter using the training images of the possible views of the target known a priori.

2. Correlation the resulting filter with the example background and calculate the value of the DC using Eq. (9).

3. If the resulting DC is greater than or equal to the desired value, the algorithm is finished. Else, continue to step 4. 
4. Locate the highest peak in the correlation output and synthesize a pattern to reject.

5. Add the synthesized pattern to the training set.

6. Go to step 1.

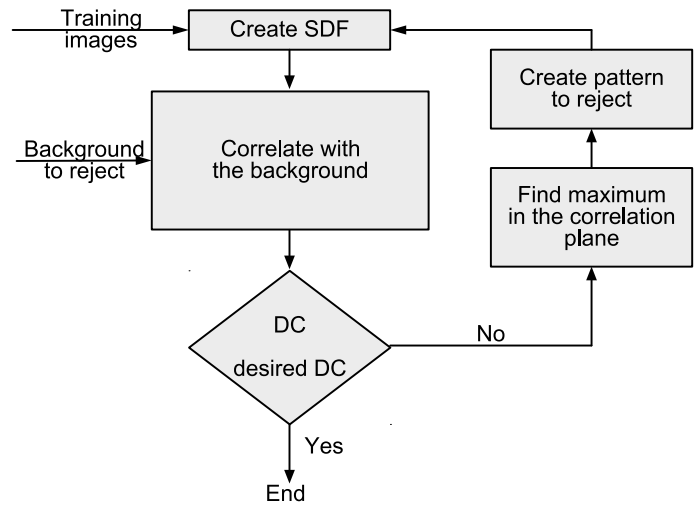

Fig. 1. Block diagram of the adaptive algorithm used for filter design

\section{Computer Simulations}

In this section we present computer simulation results. The performance of the proposed filters is evaluated in terms of DC. The size of the images used in the experiments is $256 \times 256$ pixels with intensity values in the range $[0-255]$. We use the image of the butterfly shown in Fig. 2(a) as the target. Reference images are corrupted by additive noise as shown in Fig. 2(a). The sample background used for the adaptation step of the proposed algorithm is shown in Fig. 2(b). A sample input scene is shown 2(c) with a deterministic background and additive sensor noise. The statistics of the images used are shown Table 1. To guarantee statistically correct results, 30 statistical trials of each experiment for different positions of a target and realization of random processes were carried out.

Table 1. Image statistics

\begin{tabular}{ccccc} 
Statistical parameter Mean Std. Dev. & Size & Correlation coefficient \\
\hline Target & 108 & 50 & $28 \times 44$ & - \\
Training image noise & 0 & $5-30$ & $256 \times 256$ & - \\
Training background & 112 & 40 & $256 \times 256$ & 0.9 \\
Scene background & 93 & 48 & $256 \times 256$ & 0.85
\end{tabular}




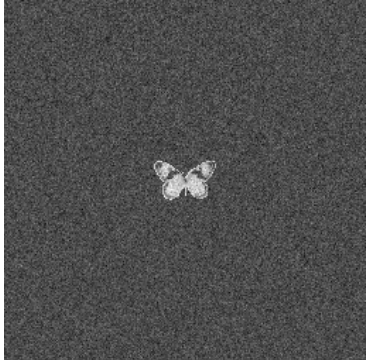

(a)

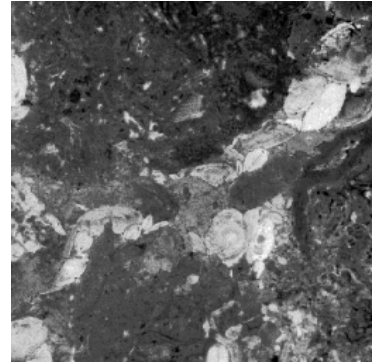

(b)

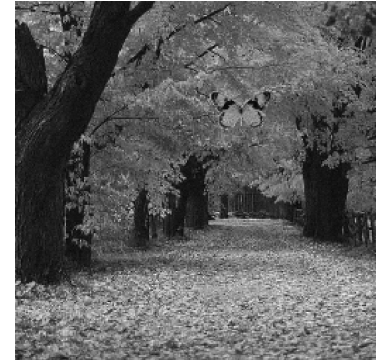

(c)

Fig. 2. (a) Sample reference image used in the experiments, (b) background used in the adaptive algorithm, (c) sample input scene with disjoint background and additive noise

Four filters are used in the experiments: the $\mathrm{GOF}_{\mathrm{AN}}$ designed using the algorithm in Ref. 10 from a noisy view of the target with rotation 0 and scale 1, and three composite filters $\mathrm{AGOF}_{\mathrm{AN}}, \mathrm{MACE}$ and $\mathrm{MACH}$. Composite filters are designed using a training set with 110 views of the target with rotations from $-20^{\circ}$ to $20^{\circ}$ in 2 degree steps and scale values from 0.8 to 1.2 in 0.1 steps. $\mathrm{AGOF}_{\mathrm{AN}}$ is the filter obtained by the proposed adaptive algorithm.

We first evaluate the performance of the filters as the standard deviation of noise in the training images is increased from 5 to 30. Simulation results are shown in Fig. 3(a). The MACH filter completely fails to detect the target due to the noise in the reference images. The MACE filter achieves poor performance even when the Std. Dev. of the noise is low. The GOF AN filter achieves good detection performance up to a Std. Dev. of 20 and degrades as the intensity of the noise increases. The $\mathrm{AGOF}_{\mathrm{AN}}$ proves to be robust to the presence of noise and achieves good detection performance even at the highest levels of noise tested.

Performance of the filters when the target in the input scene is scaled to 1.15 its original size is shown in Fig. 3(b). The MACH filter is still unable to detect the target. The performance of the MACE filter is below that of the previous case. The $\mathrm{GOF}_{\mathrm{AN}}$ filter can still detect the target but its performance is lower. This is because the $\mathrm{GOF}_{\mathrm{AN}}$ is designed to detect the target at its original size. The performance of the $\mathrm{AGOF}_{\mathrm{AN}}$ is only slightly lower but the target can still be correctly detected. We can conclude that the $\mathrm{AGOF}_{\mathrm{AN}}$ is significantly more robust to the presence of noise than the $\mathrm{GOF}_{\mathrm{AN}}$, while the MACE and MACH filters fail even with low levels of noise.

Next we analyze the performance of the filters when varying the rotation of the target. Rotation values tested are in the range $\left[-30^{\circ}, 30^{\circ}\right]$ with $3^{\circ}$ increments. The Std. Dev. of the noise in the training images is fixed at 15. Simulation results are shown in Fig. 4(a) when the target appears at 100\% its original size and in Fig. 4(b) when the target is scaled to 0.65 of its original size. The MACH filter is unable to detect the target regardless of the rotation it appears with. The MACH filter detects the target with low DC in all of the tested range. The GOF $_{\text {AN }}$ performs best when the rotation of the target is close to zero, but 


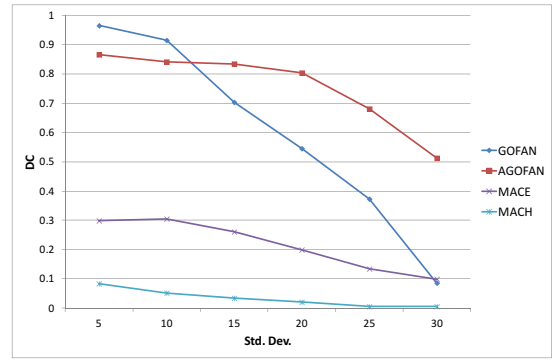

(a)

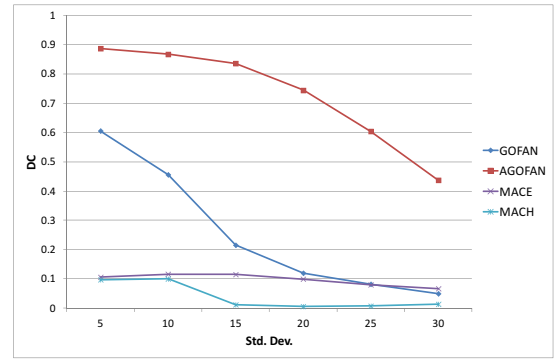

(b)

Fig. 3. Performance of filters in terms of DC while varying the Std. Dev. of the additive noise in the training images when the target scale is (a) 1.0 and (b) 1.15

degrades as the rotation changes. It is interesting to note that the performance of the $\mathrm{AGOF}_{\mathrm{AN}}$ remains almost constant even when the target is rotated beyond the range used for training. When the target is scaled to 0.65 , a value lower than those used for training, the performance of all filter degrades significantly. However, the $\mathrm{AGOF}_{\mathrm{AN}}$ can still correctly detect the target for most of the tested rotations.

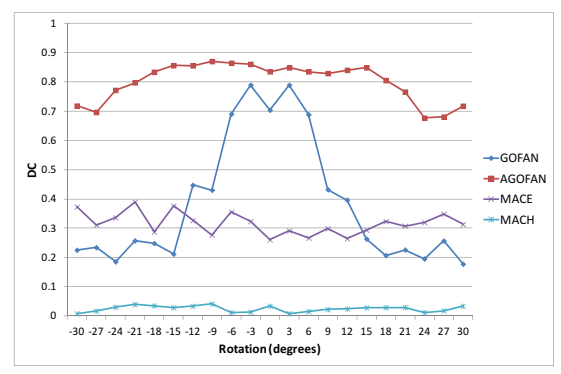

(a)

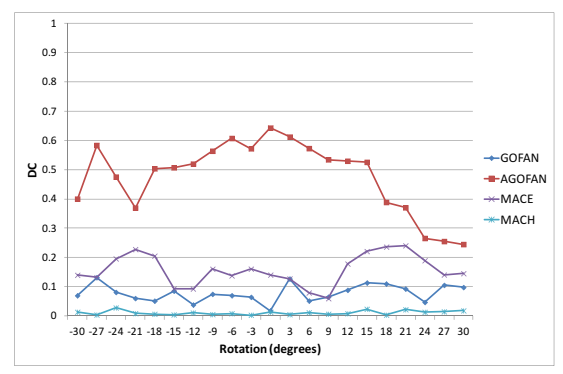

(b)

Fig. 4. Performance of filters in terms of DC while varying the rotation of the target. The target scale is (a) 1.0 and (b) 0.65 .

Finally, we analyze the performance of the filters when the varying the target scale. Scale values are in the range $[0.65,1.35]$ in 0.1 increments. Simulation results are shown in Fig. 5(a) when the target appears without rotation in the scene. The performance of the MACH filter is similar to other tested scenarios and it is not able to detect the target for any of the tested scale values. The MACE filter can detect the target but its performance is very limited. The GOF $_{\text {AN }}$ achieves its best performance when the scale of the target is 1.0 but it degrades rapidly as the target scale changes. The performance of the $\mathrm{AGOF}_{\mathrm{AN}}$ remains consistent with previous scenarios. The target is correctly detected even 
when the tested scale values are outside of the range used for filter design. Simulation results with the target rotated $15^{\circ}$ are shown in Fig. 5(b). The performance of the $\mathrm{AGOF}_{\mathrm{AN}}$ is lower than when the target is not rotated but it is still capable of correctly detecting the target for all tested values.

We can conclude that filters obtained with the adaptive algorithm are tolerant to both kinds of distortions tested, rotation and scale changes, and robust to the presence of noise. The $\mathrm{GOF}_{\mathrm{AN}}$ is robust to noise but it fails to detect the target when its appearance differs from that used for training. Finally, the performance of classic correlation filters is severely degraded when the images used for training are corrupted by additive noise, therefore, using an algorithm that accounts for noise is more appropriate.

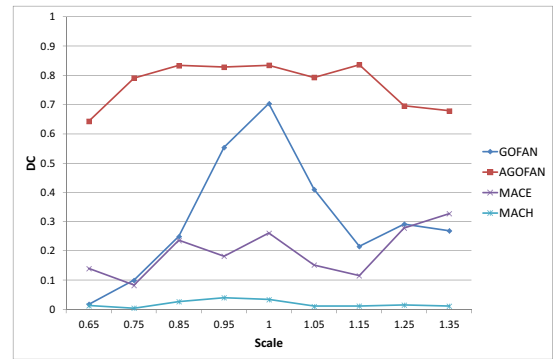

(a)

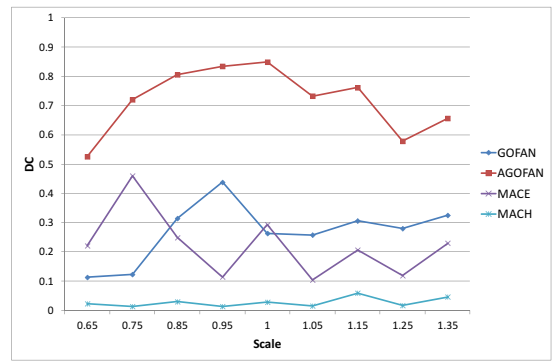

(b)

Fig. 5. Performance of filters in terms of DC while varying the scale of the target. The target rotation is (a) 0 and (b) $15^{\circ}$.

\section{Conclusions}

In this work we proposed adaptive correlation filters for detecting a target in a nonoverlapping scene. The training images used for filter design are assumed to be corrupted by additive noise. Filters are composed as a linear combination of optimum filter impulse responses and matched filters. The appearance and shape of each known view of the object of interest are estimated from the noisy training images. An iterative procedure is used to adapt the composite filter to a typical background where detection will be performed. Computer simulation results show that the proposed composite filters achieve good detection performance terms even when the training images contain additive noise. Moreover, the proposed filters outperform classic composite filters used for distortion invariant object detection when noise in the reference image increases and distortions in the input scene are outside the range used during training.

\section{References}

1. Javidi, B.: Real-Time Optical Information Processing. Academic Press, New York (1994) 
2. Kumar, B.V.K.V., Mahalanobis, A., Juday, R.: Correlation pattern recognition. Cambridge Univ. Press, New York (2005)

3. Réfrégier, P., Goudail, F.: Statistical Image Processing Techniques for Noisy Images: An Application-Oriented Approach. Kluwer Academic Publishers, New York (2003)

4. Yaroslavsky, L.P.: The theory of optimal methods for localization of objects in pictures. In: Wolf, E. (ed.) Progress in Optics, pp. 145-201. Elsevier, Amsterdam (1993)

5. Kober, V., Campos, J.: Accuracy of location measurement of a noisy target in a nonoverlapping background. Journal of the Optical Society of America A 13(8), 1653-1666 (1996)

6. Kumar, B.V.K.V., Dickey, F.M., DeLaurentis, J.M.: Correlation filters minimizing peak location errors. Journal of the Optical Society of America A 9(5), 678-682 (1992)

7. VanderLugt, A.: Signal detection by complex spatial filtering. IEEE Transactions on Information Theory 10(2), 139-145 (1964)

8. Javidi, B., Wang, J.: Design of filters to detect a noisy target in nonoverlapping background noise. Journal of the Optical Society of America A 11(10), 2604-2612 (1994)

9. Aguilar-González, P.M., Kober, V.: Design of correlation filters for pattern recognition with disjoint reference image. Optical Engineering 50, 117201 (2011)

10. Aguilar-González, P.M., Kober, V.: Design of correlation filters for pattern recognition using a noisy reference. Optics Communications 285(5), 574-583 (2012)

11. Kumar, B.V.K.V.: Tutorial survey of composite filter designs for optical correlators. Applied Optics 31(23), 4773-4801 (1992)

12. Kerekes, R.A., Kumar, B.V.K.V.: Selecting a composite correlation filter design: a survey and comparative study. Optical Engineering 47(6), 067202.1-067202.18 (2008)

13. Casasent, D.: Unified synthetic discriminant function computational formulation. Applied Optics 23(10), 1620-1627 (1984)

14. Mahalanobis, A., Kumar, B.V.K.V., Casasent, D.: Minimum average correlation energy filters. Applied Optics 26(17), 3633-3640 (1987)

15. Mahalanobis, A., Kumar, B.V.K.V., Song, S., Sims, S.R.F., Epperson, J.F.: Unconstrained correlation filters. Applied Optics 33(17), 3751-3759 (1994)

16. Díaz-Ramírez, V.H., Kober, V.: Adaptive phase-input joint transform correlator. Applied Optics 46(26), 6543-6551 (2007)

17. Díaz-Ramírez, V.H., Kober, V., Álvarez-Borrego, J.: Pattern recognition with an adaptive joint transform correlator. Applied Optics 45(23), 5929-5941 (2006)

18. González-Fraga, J., Kober, V., Álvarez-Borrego, J.: Adaptive synthetic discriminant function filters for pattern recognition. Optical Engineering 45, 057005.1$057005.10(2006)$

19. Ramos-Michel, E.M., Kober, V.: Design of correlation filters for recognition of linearly distorted objects in linearly degraded scenes. Journal of the Optical Society of America. A 24(11), 3403-3417 (2007)

20. Ramos-Michel, E.M., Kober, V.: Adaptive composite filters for pattern recognition in linearly degraded and noisy scenes. Optical Engineering 47(4), 047204.1047204.7 (2008)

21. Yaroslavsky, L.P.: The theory of optimal methods for localization of objects in pictures. In: Wolf, E. (ed.) Progress in Optics, pp. 145-201. Elsevier (1993) 